\title{
BMJ Open Assessment of dialysis initiation by a fuzzy mathematics equation (ADIFE): a study protocol for a randomised controlled trial
}

\author{
Jilin Chen, ${ }^{1,2}$ Ying Liu, ${ }^{1,2}$ Xiangmei Chen, ${ }^{3}$ Xuefeng Sun, ${ }^{3}$ Wei Li, ${ }^{4}$ Wang Yang, ${ }^{4}$ \\ Ping Li, ${ }^{3}$ Ximing Sun, ${ }^{5}$ Degang Wang, ${ }^{5}$ Hongli Jiang, ${ }^{6}$ Wei Shi, ${ }^{7}$ Wenhu Liu, ${ }^{8}$ \\ Ping Fu, ${ }^{9}$ Xiaoqiang Ding, ${ }^{10}$ Ming Chang, ${ }^{11}$ Shuxin Liu, ${ }^{11}$ Xiao Yang, ${ }^{12}$ Ning Cao, ${ }^{13}$ \\ Menghua Chen, ${ }^{14}$ Zhaohui Ni, ${ }^{15}$ Jing Chen, ${ }^{16}$ Shiren Sun, ${ }^{17}$ Xinling Liang, ${ }^{18}$ \\ Huimin Wang, ${ }^{19}$ Yani He ${ }^{20}$ Bihu Gao, ${ }^{21}$ Jianqin Wang, ${ }^{22}$ Lirong Hao, ${ }^{23}$ Jian Liu, ${ }^{24}$ \\ Suhua Li, ${ }^{24}$ Qiang He,${ }^{25}$ Hongmei Liu, ${ }^{26} \mathrm{Na} \mathrm{Yi},{ }^{26}$ Fengmin Shao, ${ }^{27}$ Jundong Jiao, ${ }^{28}$ \\ Yuhuan Ma, ${ }^{29} \mathrm{Li}$ Yao, ${ }^{30}$ Yi Sun, ${ }^{31}$ Detian Li, ${ }^{32}$ Lynda Szczech, ${ }^{33}$ Ming Fang, ${ }^{2}$ \\ Zach Odeh, ${ }^{1}$ Hongli Lin ${ }^{1,2}$
}

To cite: Chen J, Liu Y, Chen $\mathrm{X}$, et al. Assessment of dialysis initiation by a fuzzy mathematics equation (ADIFE): a study protocol for a randomised controlled trial. BMJ Open 2019;9:e023162. doi:10.1136/ bmjopen-2018-023162

- Prepublication history and additional material for this paper are available online. To view these files, please visit the journal online (http://dx.doi. org/10.1136bmjopen-2018023162).

$\mathrm{JC}$ and YL contributed equally.

$\mathrm{JC}$ and $\mathrm{YL}$ are joint first authors.

Received 24 March 2018 Revised 08 May 2019 Accepted 03 July 2019

Check for updates

(c) Author(s) (or their employer(s)) 2019. Re-use permitted under CC BY-NC. No commercial re-use. See rights and permissions. Published by BMJ.

For numbered affiliations see end of article.

Correspondence to Professor Hongli Lin linhongli@vip.163.com

\section{ABSTRACT}

Introduction Starting dialysis early or late both result in a low quality of life and a poor prognosis in patients undergoing haemodialysis. However, there remains no consensus on the optimal timing of dialysis initiation, mainly because of a lack of suitable methods to assess variations in dialysis initiation time. We have established a novel equation named DIFE (Dialysis Initiation based on Fuzzy-mathematics Equation) through a retrospective, multicentre clinical cohort study in China to determine the most suitable timing of dialysis initiation. The predictors of the DIFE include nine biochemical markers and clinical variables that together influence dialysis initiation. To externally validate the clinical accuracy of DIFE, we designed the assessment of DIFE (ADIFE) study as a prospective, open-label, multicentre, randomised controlled trial to assess the clinical outcomes among patients who initiate dialysis in an optimal start dialysis group and a late-start dialysis group, based on DIFE.

Methods and analysis A total of 388 enrolled patients with end-stage renal disease will be randomised 1:1 to the optimal start dialysis group, with a DIFE value between 30 and 35 , or the late-start dialysis group, with a DIFE value less than 30 , using the Randomization and Trial Supply Management system. Participants will be assessed for changes in signs and symptoms, dialysis mode and parameters, biochemical and inflammatory markers, Subjective Global Assessment, Kidney Disease Quality of Life Short Form, Cognitive Assessment, medical costs, adverse events and concomitant medication at baseline, predialysis visiting stage and postdialysis visiting stage, every 12-24 weeks. The following data will be recorded on standardised online electronic case report forms. The primary endpoint is 3-year all-cause mortality. The secondary endpoints include non-fatal cerebrocardiovascular events, annual hospitalisation rate, quality of life, medical costs and haemodialysis related complications.
Strengths and limitations of this study

- We established a novel and quantifiable equation (dialysis initiation based on fuzzy-mathematics equation (DIFE)), containing nine laboratory and clinical parameters that together influence the timing of dialysis initiation, which showed significant advantages to assess the timing of dialysis initiation compared with the estimated glomerular filtration rate alone.

- This is the first prospective, randomised controlled study to assess the timing of initiation of dialysis based on DIFE in patients with end-stage renal disease (ESRD).

- The study will provide acceptability and feasibility data for optimal dialysis initiation based on DIFE to avoid early and late-start dialysis in patients with ESRD.

- Participants will be recruited from 25 haemodialysis centres in China, which may introduce sample selection bias.

- The haemodialysis centres do not all use the same dialysers to treat the participants.

Ethics and dissemination Ethical approval was obtained from the Ethics Committee of the First Affiliated Hospital of Dalian Medical University China (registration no: YJ-KY-2017-119) and the ethics committees of all participating centres. The final results of the ADIFE trial will be presented to the study sponsor, clinical researchers and the patient and public involvement reference group. Findings will be disseminated through peer-reviewed journals, Clinical Practice Guidelines and at scientific meetings.

Trial registration number ClinicalTrial.gov. Registry (NCT03385902); pre-results. 


\section{INTRODUCTION}

The growing prevalence and incidence of end-stage renal disease (ESRD) represents a global health challenge. ${ }^{1}$ Haemodialysis is the main treatment for patients with ESRD, and its start time has a significant effect on patient survival. ${ }^{2-4}$ Late or early start of dialysis can negatively affect the quality of life and survival prognosis of patients, and this suboptimal timing of dialysis results in an increased economic burden for families and society. ${ }^{5-7}$ Therefore, determining and implementing the optimal time to commence dialysis could improve a patient's quality of life by relieving their uraemic symptoms, decreasing their risk of early death and by reducing medical care costs. ${ }^{4}$ However, there is still no consensus on the optimal timing of dialysis initiation for patients with ESRD, and it is unknown what is exactly the optimal timing of dialysis is associated with better outcomes. Several observational studies found that an earlier start of dialysis was associated with improved survival and better prognosis. ${ }^{589}$ However, certain cohort studies and a randomised controlled trial (the Initiating Dialysis Early and Late (IDEAL) study) have shown that patients receiving early initiation of dialysis were at risk of poor survival and that late initiation of dialysis was associated with lower risk of mortality and improved survival. ${ }^{10-13}$ However, these findings are controversial mainly because of the inefficient or outdated methods of assessing dialysis timing used. All of the above studies used the creatinine-based estimated glomerular filtration rate (eGFR), a value whose specificity is affected by nutritional status and muscle mass, and is calculated by either the Modified Diet in Renal Disease equation or the Cockcroft-Gault equation. ${ }^{14}$ Studies have shown that some clinical factors, such as older age, volume overload, malnutrition, diabetes and heart failure, strongly influence the timing of dialysis initiation. ${ }^{516-18}$ Therefore, the Kidney Disease Outcomes Quality Initiative Clinical Practice Guideline for Hemodialysis Adequacy recommend that the decision to initiate maintenance dialysis should be based primarily on the assessment of specific complications of kidney disease, including signs and symptoms of uraemia, protein-energy wasting, metabolic abnormalities and volume overload rather than based on the eGFR alone. ${ }^{19}{ }^{20}$ Deviation from an empirical decision to an assessment of varying clinical conditions inevitably leads to a lack of consensus because of clinicians' subjective judgements, which can lead to a suboptimal decision regarding early or late initiation of dialysis.

Thus, our research team established a novel equation to determine the optimal timing, called dialysis initiation based on a fuzzy mathematical method (DIFE), which was derived from a previous multicentre, retrospective cohort study with large-scale samples. ${ }^{21}$ The DIFE includes nine parameters: sex, age, blood urea nitrogen, serum creatinine, haemoglobin, serum albumin, serum phosphorus, heart failure condition and diabetes condition, which effectively combines subjective clinical variables with objective biochemical markers for dialysis initiation decision- making. The DIFE study showed that the 3-year dialysis mortality of patients in the optimal start dialysis group (DIFE value of 30 to 35) was markedly lower $(8.38 \%)$ than the late-start dialysis group (DIFE value less than 30 ) of $19.4 \%$. Moreover, receiver operating characteristic curve analysis indicated that the area under the curve for the prediction of a 3-year death during dialysis initiation assessed by the DIFE was significantly higher than that predicted by eGFR $(0.70$ vs $0.55, \mathrm{p}<0.01) .^{21}$ Therefore, the DIFE is more accurate and effective to assess the timing of haemodialysis initiation than eGFR alone. Furthermore, the DIFE equation is convenient for popularisation and application because it transforms subjective clinical factors into objective parameters, which will be especially appealing to non-nephrologists and doctors in primary hospitals. DIFE may become the new standard in the assessment of the timing of dialysis, replacing eGFR. To further evaluate the predictive ability and clinical accuracy of DIFE, we designed a prospective, multicentre randomised controlled trial, involving 25 hospitals located in different regions in China, to assess clinical outcomes of patients with ESRD placed in optimal or late-start dialysis cohorts on the basis of DIFE. The aims of the trial are to assess the effect of the optimal and late-start dialysis, based on DIFE, using the 3-year mortality rate, hospitalisation, morbidity, quality of life and medical costs of patients receiving haemodialysis. We believe that the assessment of DIFE (ADIFE) study will provide clinical evidence for the optimal time to start dialysis in patients with ESRD based on DIFE.

\section{METHODS AND ANALYSIS \\ Study design}

The ADIFE study is a prospective, multicentre, randomised, controlled, open-label trial in patients with ESRD. Patients will be divided into an 'optimal-start dialysis' group, with DIFE value between 30 and 35, and a 'late-start dialysis' group, with a DIFE value less than 30 . The study will be implemented in 25 dialysis centres, covering the seven administrative regions in China (North China, East China, South Central, Northeast, Southwest and Northwest). Each participating centre has a systemic follow-up procedure for the participants with chronic kidney disease (CKD) and can provide predialysis care including preparation of vascular access in patients approaching haemodialysis.

Participants will be followed up at baseline, at the predialysis visiting stage every 12 weeks and at the postdialysis visiting stage every 12 or 24 weeks. A flow diagram of the whole trial is shown in figure 1. The protocol of the ADIFE study was designed according to the Standard Protocol Items: Recommendations for Interventional Trials (SPIRIT) reporting guidelines. ${ }^{22}$

\section{Participants}

Inclusion criteria

Participants will be enrolled the study if they meet all the following requirements: 


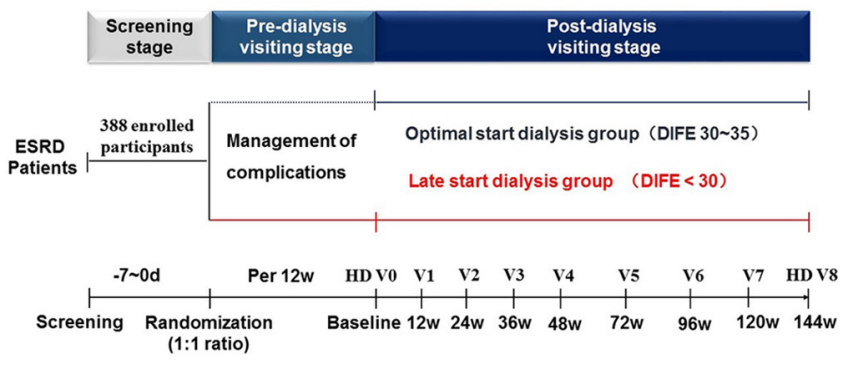

Figure 1 Trial flow diagram of the ADIFE study. The whole trial flow, including the screening stage within 7 days, predialysis visiting stage and postdialysis visiting stage of visit 0 to visit 8 . Enrolled subjects will be randomised $1: 1$ to the optimal-start dialysis group with a DIFE value between 30 and 35 or to the late-start dialysis group with a DIFE value less than 30 . Participants will be followed up at baseline (visit 0 ), predialysis visiting stage every 12 weeks and postdialysis visiting stage every 12 or 24 weeks. ESRD, end-stage renal disease; DIFE, dialysis initiation based on fuzzy-mathematics equation.

1. Adults age between 18 and 75 years old.

2. Chronic kidney disease with an eGFR (calculated by the Chronic Kidney Disease Epidemiology Collaboration (CKD-EPI) equation ${ }^{23}$ ) of less than $15 \mathrm{~mL} /$ $\mathrm{min} / 1.73 \mathrm{~m}^{2}$ and a DIFE value between 30 and 35 .

3. Expected to commence maintenance haemodialysis.

4. Agreeable to randomisation.

Exclusion criteria

Participants will be excluded if they meet one of the following items:

1. Acute kidney injury (AKI) or AKI on CKD.

2. Having a primary disease comprising systemic lupus erythematosus or systemic vasculitis.

3. Have received, or are planning to receive, a kidney transplantation or peritoneal dialysis.

4. Recently diagnosed cancer that is likely to impact on survival (except for cured cancer or cancer in remission for over 5 years, after radical resection of the basal cell carcinoma or squamous carcinoma of skin or carcinoma in situ of any part of the body).

5. Hepatocirrhosis.

6. Positive test for HIV, hepatitis B virus (HBV) antigen or antihepatitis $\mathrm{C}$ virus (HCV) antibody.

7. Acute infectious disease within 1 month.

8. Poor lifestyle choice that is difficult to withdraw from, such as alcohol abuse.

9. Poor compliance.

10. Being pregnant, nursing or planning for pregnancy.

11. Life expectancy less than 1 year.

12. Other cases in which the investigator confirms that they should not enrol in the study.

\section{Sample size}

The sample size estimate is mainly based on the primary endpoint of the 3-year all-cause mortality from the previous retrospective cohort DIFE study, which showed that the 3-year mortality of the optimal start dialysis group was $8.38 \%$ and that of the late start dialysis group was $19.4 \% .{ }^{21}$ Using PASS V.15 of the Power and Sample Size Calculation program (NCSS, LLC), we estimated a sample size of 154 per group (assuming a type I error rate of $5 \%$ with $80 \%$ power, two-sided test, with $\mathrm{p}<0.05$ considered statistically significant). Assuming that $20 \%$ of the participants would withdraw or drop out, the target sample size was estimated as 388 participants, meaning that 194 participants in each group will be recruited.

\section{Randomisation and allocation}

Independent biostatisticians with no relationship to the data management or the data statistical analysis team will use the SAS V.9.2 software (V.9.2; SAS Institute) stratified by site to generate random numbers according to the block randomisation method. The investigators will allocate the random numbers to eligible participants, assigned 1:1 to the optimal-start dialysis group or the latestart dialysis group, according to the enrolment sequence. Randomisation allocation and random numbers will be sent by automated email to the non-blind investigators performing the randomisation using their unique user name and password on the internet-based randomisation service of a Randomization and Trial Supply Management system.

\section{Patient and public involvement}

A patient and public involvement (PPI) reference group, comprising dialysis patients, their partners, carers and representatives from voluntary sectors will be formed. The PPI reference group will meet quarterly throughout the duration of the programme. Group members have been consulted at all stages of the work leading to this proposal and have provided valuable insight and advice. The trial was designed in partnership with the PPI to help maximise patient benefits. Our PPI representatives have materially influenced decisions on the study population, promotion and recruitment. They will also continue to contribute throughout this pilot study in terms of reviewing documentation for ethics approval, reading reports and contributing to dissemination activities. We will also present the final results of the ADIFE trial to the PPI.

\section{Treatment}

All participants will receive regular treatment as usual, which includes regular dietary advice, anaemia and CKDmineral and bone disorder management, blood pressure, and volume control, as recommended by the Kidney Disease Improving Global Outcomes (KDIGO) guidelines and the Chinese Hemodialysis Adequacy guidelines. ${ }^{1924-27}$ Different types of vascular access, including temporary venous catheters, arteriovenous fistulas and artificial blood vessels are permitted for use in all participants. The use of such catheters will be based only on clinical requirements. Each participating centre has been advised to consider early-access creation in each participant to avoid delay in the subsequent haemodialysis treatment. 
All participants will undergo haemodialysis treatment using capacity control dialysis machines, bicarbonate dialysate, a blood flow volume of $200-300 \mathrm{~mL} / \mathrm{min}$ and a disposable high-flux or low-flux dialyser with membrane area of $1.3-1.6 \mathrm{~m}^{2}$. The dialysis dose is 4 hours per treatment, performed two or three times per week; and the recommended single pool $\mathrm{Kt} / \mathrm{V}(\mathrm{spKt} / \mathrm{V})$ is more than 1.2. ${ }^{19} 2728$ However, despite the existence of dialysis management guidelines in China, there is still a potential for treatment variation between the participating centres.

\section{Intervention}

Participants allocated to the 'optimal-start dialysis' group will commence dialysis when their DIFE values are between 30 and 35 . Participants allocated to the 'late-start dialysis' group will be monitored based on the changes in DIFE values in the predialysis visiting stage every 12 weeks until their DIFE values are less than 30, and then dialysis will commence. Participants allocated to the 'late-start dialysis' group will be able to commence dialysis earlier based on the recommendation of their caring physician, although they should have a DIFE value of no less than 30 .
For instance, participants showing obvious uraemia symptoms, volume overload and hyperkalaemia, for which the reasons for early initiation of dialysis will be recorded; this will allow for a subsequent analysis of actual DIFE at the dialysis start time.

\section{Outcome measurement}

All enrolled participants will be followed up until death or until 144 weeks after the last patient is randomised. Participants in the 'late-start dialysis' group will be assessed every 12 weeks in the predialysis visiting stage. During the postdialysis visiting period, data will be collected every 12 weeks in the first year of follow-up and every 24 weeks in the next 2 years of follow-up. The detailed follow-up items in different visiting stage are shown in table 1.

Comprehensive demographic data (age, gender, ethnicity, height, weight, education, employment, causes of ESRD, medical history, presence of comorbid conditions) will be collected for all participants at baseline. Virology examination (HBV antigen, HCV antibody, HIV antibody, syphilis antibody) and human urine chorionic gonadotropin will be tested in the screening stage.

Table 1 The follow-up items in different visiting stage of ADIFE study

\begin{tabular}{|c|c|c|c|}
\hline & & & Postdialysis visiting stage \\
\hline Follow-up items & Screening stage & Predialysis visiting stage & V0-V8 (0-144w) \\
\hline Signed informed consent form & $\sqrt{ }$ & - & - \\
\hline Inclusion and exclusion criteria & $\sqrt{ }$ & - & - \\
\hline Demographic data & $\sqrt{ }$ & - & - \\
\hline Vital signs, physical examination & $\sqrt{ }$ & $\sqrt{ }$ & $\sqrt{ }(\mathrm{V} 0-\mathrm{V} 8)$ \\
\hline Urine HCG & $\sqrt{ }$ & - & - \\
\hline Virology examination & $\sqrt{ }$ & - & - \\
\hline Blood routine test & $\sqrt{ }$ & $\sqrt{ }$ & $\sqrt{ }(\mathrm{V} 0-\mathrm{V} 8)$ \\
\hline $\begin{array}{l}\text { BUN, Scr, eGFR, Alb, } \\
\text { electrolytes, }\end{array}$ & $\sqrt{ }$ & $\sqrt{ }$ & $\sqrt{ }(\mathrm{V} 0-\mathrm{V} 8)$ \\
\hline $\begin{array}{l}\text { ALT, AST, T-BIL, blood glucose, } \\
\text { serum lipid, serum iron }\end{array}$ & - & - & $\sqrt{ }(\mathrm{V} 0, \mathrm{~V} 4, \mathrm{~V} 6, \mathrm{~V} 8)$ \\
\hline PTH, ferritin & - & - & V (V0, V2, V4-V8) \\
\hline $\begin{array}{l}\text { Hs-CRP, IL-6, IL-10, } \\
\text { TNF- } \alpha, \beta_{2}-\mathrm{MG}\end{array}$ & - & - & $\sqrt{ }(\mathrm{V} 0, \mathrm{~V} 2, \mathrm{~V} 4-\mathrm{V} 8)$ \\
\hline KDQoL-SF & - & - & $\sqrt{ }(\mathrm{V} 0, \mathrm{~V} 4, \mathrm{~V} 6, \mathrm{~V} 8)$ \\
\hline MoCA & - & - & $\sqrt{ }(\mathrm{V} 0, \mathrm{~V} 2, \mathrm{~V} 4-\mathrm{V} 8)$ \\
\hline SGA & - & - & V (V0, V2, V4-V8) \\
\hline Vascular access & - & - & $\sqrt{ }(\mathrm{V} 0-\mathrm{V} 8)$ \\
\hline Medical costs & - & $\sqrt{ }$ & $\sqrt{ }(\mathrm{V} 0-\mathrm{V} 8)$ \\
\hline Complications related to dialysis & - & - & $\sqrt{ }(\mathrm{V} 0-\mathrm{V} 8)$ \\
\hline AEs, SAEs & - & $\sqrt{ }$ & $\sqrt{ }(\mathrm{V} 0-\mathrm{V} 8)$ \\
\hline Concomitant medications & - & $\sqrt{ }$ & $\sqrt{ }(\mathrm{V} 0-\mathrm{V} 8)$ \\
\hline
\end{tabular}

' $\sqrt{ }$ ' represents selected follow-up items; '-' represents not-selected follow-up items.

AEs, adverse events; ALB, albumin; ALT, alanine transaminase; AST, aspartate transaminase; BUN, blood urea nitrogen;eGFR, estimated glomerular filtration rate; HCG, human chorionic gonadotropin; Hs-CRP, high sensitive C-reactive protein; IL, interleukin; KDQoL-SF, Kidney Disease Quality of Life Short Form; $\beta_{2}-M G, \beta_{2}$-microglobulin; MoCA, Montreal Cognitive Assessment; PTH, parathyroid hormone; SAEs, serious adverse events; Scr, serum creatinine;SGA, Subjective Global Assessment; T-BIL, total bilirubin; TNF- $\alpha$, tumour necrosis factor alpha. 
Vital signs, including temperature, heart rate, respiratory rate, and non-invasive blood pressure will be monitored at each follow-up. Biochemical indexes, including blood cell count (red cells, white cells and platelets), haemoglobin, blood urea nitrogen (BUN), serum creatinine ( $\mathrm{Scr}$ ), eGFR, electrolytes (serum sodium, serum potassium, serum chloride, serum calcium and serum phosphate), alanine transaminase, glutamic-oxaloacetic transaminease, total bilirubin, blood glucose, serum lipid, serum iron, parathyroid hormone (PTH) and ferritin will be tested every 12 or 24 weeks in each participating centre. Inflammatory biomarkers, including high-sensitivity C-reactive protein (hs-CRP), interleukin (IL)-6, IL-10, tumour necrosis factor alpha and $\beta_{2}$ microglobulin will be tested every 24 weeks by the central laboratory in the Kidney Disease Research Institute of Dalian Medical University. Nutritional status, assessed using Subjective Global Assessment (SGA) ${ }^{29}$ and the serum albumin level,will be assessed every 24 weeks. Quality of life will be measured using the well-validated Kidney Disease Quality of Life Short Form ${ }^{30} 31$ every 48 weeks; and cognitive function will be assessed using the Montreal Cognitive Assessment ${ }^{32}$ every 24 weeks. The use of concomitant medications, including calcium channel blockers, statins, ACE inhibitors, angiotensin receptor blockers and erythropoiesis stimulating agents will be recorded at baseline and during the follow-up period. Medical costs will be recorded in the predialysis visiting stage and postdialysis visiting stage using both the questionnaire and medical insurance records of the participants, including dialysis-related costs, hospitalisation-related costs and outpatient costs of comorbidities treatment.

All the above-mentioned data will be recorded on standardised online electronic case report forms (eCRFs) based on an electronic data acquisition system. All adverse events (AEs) will be recorded on the eCRFs on specific pages reserved for this purpose. Serious adverse events (SAEs) are defined as death, life-threatening illness, hospitalisation (or prolongation of initial hospitalisation), causing disability or permanent damage, a congenital anomaly or a birth defect. Completed eCRFs will be entered into a secured central database for independent quality control and centralised analysis.

\section{Endpoint measurements}

The primary endpoint is the 3-year all-cause mortality following randomisation to the 'optimal-start dialysis' or 'late-start dialysis' groups. Secondary endpoints include cerebrocardiovascular events (non-fatal myocardial infarction, non-fatal stroke, transient ischaemic attack, new-onset angina, acute heart failure or severe arrhythmia requiring hospitalisation), infectious complications, haemodialysis complications (including changes of vascular access, vascular access related infection, fluid and electrolyte disorders and cognitive dysfunction), annual hospitalisation (proportion of participants admitted to hospital every year), quality of life, nutrition assessment, cognitive dysfunction and medical costs.

\section{Statistical analysis}

For all baseline and outcome variables, the number of available measurements and the number of missing values will be recorded. All analyses will be conducted according to the intention-to-treat (ITT) principle, ${ }^{33}$ that is, all randomised patients will be analysed in the groups to which they were originally allocated, and non-compliance with treatment and other violations of the protocol will be measured and reported as an ITT effect estimate. We also will perform inverse probability weighting to adjust the selection bias due to attrition. ${ }^{34}$ Continuous variables will be checked for normal distribution and will be presented as the mean and $\mathrm{SD}$ or median and $\mathrm{IQR}$ as appropriate. Comparisons of continuous variables will be performed using Student's t-test for normally distributed variables and the Mann-Whitney $\mathrm{U}$ test for non-normally distributed variables. Categorical variables will be presented as numbers and percentages and analysed using the $\chi^{2}$ test. The primary outcome of 3-year all-cause mortality will be compared between the optimal start and late start dialysis groups using the Cochran-Mantel-Haenszel procedure, adjusting for centre. A logistics regression model will be performed to adjust for potential confounders, such as age, sex, body mass index, urine volume, SGA, BUN, Scr, haemoglobin, serumebumin, serum phosphorus, PTH, ferritin, Hs-CRP, IL-6, IL-10, TNF- $\alpha, \beta_{2}-\mathrm{MG}$, cause of ESRD, vascular access, comorbidity, complication of dialysis and dialysis mode. All analyses will be performed using SAS V.9.2 (SAS Institute) by the team of statisticians at the Fuwai Hospital, China. All reported p values will be two sided, and $\mathrm{p}$ values less than 0.05 will be considered significant.

\section{ETHICS AND DISSEMINATION \\ Ethics}

Ethical approval for the study protocol was obtained from the ethics committees of the First Affiliated Hospital of Dalian Medical University (Registration No: YJ-KY-2017119 ) and the ethics committees of all participating centres (see online supplementary file). The study was registered on 29 December 2017 at the ClinicalTrial.gov (NCT03385902).

\section{Informed consent and withdrawal from the study}

Each participant or their authorised surrogates will sign an informed consent form. The process of informed consent will be in accordance with the Declaration of Helsinki. Participants will be fully informed about the ADIFE study by the investigators, and will be able to discuss the trial process with their nephrologists and contact the investigator directly to request further information. Participants and authorised surrogates will receive the related materials of informed consent. Participants will be informed of their right to withdraw from the study, either at their own request or at the discretion of the investigator, at any time without their care being affected in any way. 


\section{Dissemination plan}

Survey data will be exported directly from the eCRFs as a text file and imported in electronic form for scoring and analysis using statistics software. A detailed database will track participants' progress through the trial, including the scheduling of assessments and reminders to complete assessments. Detailed strategies, including phone or text message reminders, will be used to remind participants about upcoming assessments. All members of the research team and other associated personnel will have access to the final trial dataset in both identified and reidentifiable forms.

Printed data will be stored in locked filing cabinets, accessible only to the research team. Electronic data will be stored on password-protected computers or servers that are only accessible to the research team. All paper and electronic records will be retained and disposed of in accordance with the requirements of the Criteria for the Quality Control of Clinical Trial from Drugs China Food and Drug Administration.

The results from the outcome measures will not be presented in a way that compromises the confidentiality of the participants. Descriptions of participants will not allow identification of individual participants, and individual results and individual names will not be revealed. Final reports and publications will only comprise aggregated results. At completion of the study, participants will receive a plain text summary of the study results in Chinese. Scientific reports of the main outcomes, secondary outcomes and process evaluation will be submitted to an international peer-reviewed journal. The results will also be presented at national and international conferences relevant to the subject fields.

\section{Data management}

All information concerning the participants will be recorded on standardised online eCRFs, which will be anonymised and saved on password-protected computers. The data monitoring committee, which will be independent from the sponsor and any other competing interests, will meet twice yearly to review the efficacy and safety data.

\section{Oversight committees}

A Trial Steering Committee has been set up and will include an independent chairperson, 25 independent members, and the study investigators.

\section{Safety monitoring}

AEs will be closely monitored. These are events that are likely to affect the safety or physical or mental integrity of the participants in the trial to a significant degree. SAEs must be reported to the sponsor (the First Affiliated Hospital of Dalian Medical University, China) and the State Food and Drug Administration promptly, by fax or telephone, by the investigators, followed by a written report within 24 hours. The sponsor will be notified immediately of any case where the above definition applies during the trial.

\section{Discussion}

The timing of dialysis initiation is a risk factor that affects the prognosis of patients with ESRD. However, the optimal timing of dialysis initiation remains unclear. Some studies showed that an early start of dialysis was associated with a lower risk of mortality, ${ }^{5}$ whereas others studies indicated either a survival advantage of late-start dialysis $^{3111235}$ or comparable mortality risk between earlystart and late-start dialysis. ${ }^{36}{ }^{37}$ The IDEAL study indicated that using eGFR as the primary guide for when to start dialysis should probably be abandoned in a patient with progressive advanced CKD. ${ }^{10}$

The novel DIFE score integrates subjective clinical variables of uraemic signs and symptoms with objective biochemical markers beyond serum creatinine and eGFR to assess the timing of dialysis initiation in patients with ESRD, providing an individualised, effective and convenient tool for dialysis initiation decision-making. The results of ADIFE study will provide evidence to evaluate the accuracy and efficacy of DIFE and should indicate optimal timing of dialysis initiation for patients with ESRD approaching the need for maintenance dialysis. For some nephrologists and non-nephrologists with less training who may not know how to interpret laboratory values and clinical signs, a formula to calculate when to start dialysis could help them to deliver safe care.

The DIFE formula was established via a previous retrospective multicentre cohort study using data from patients receiving haemodialysis, and the ADIFE study will further assess the clinical accuracy and applicability of DIFE to guide the timing of haemodialysis initiation. Therefore, the ADIFE study will exclude participants awaiting peritoneal dialysis and transplantation; however, we have already planned to assess the applicability of DIFE to guide the timing of dialysis for patients undergoing peritoneal dialysis in another clinical study.

\section{Trial status}

Recruitment has commenced using digital social media networks and print-based advertising nationwide in April 2018. Completion of recruitment is expected in April 2020. The study will be completed by December 2023 .

\section{Author affiliations}

${ }^{1}$ Graduate School of Dalian Medical University, Dalian, , China

${ }^{2}$ Department of Nephrology, The First Affiliated Hospital of Dalian Medical University. Kidney Research Institute of Dalian Medical University, Dalian, , China

${ }^{3}$ Department of Nephrology, Chinese PLA General Hospital, Chinese PLA Institute of Nephrology, State Key Laboratory of Kidney Diseases, National Clinical Research Center for Kidney Diseases, Beijing Key Laboratory of Kidney Disease Research, Beijing, China

${ }^{4}$ Medical Research \& Biometrics Center, Fuwai Hospital, National Center for Cardiovascular Disease, Chinese Academy of Medical Sciences and Peking Union Medical College, Beijing, China

${ }^{5}$ School of Control Science and Engineering, Dalian University of Technology, Dalian, China

${ }^{6}$ Blood Purification Center, The First Affiliated Hospital of Xi'an Jiaotong University, Xi'an, China 
${ }^{7}$ Division of Nephrology, Guangdong General Hospital, Guangdong Academy of Medical Sciences, Guangzhou, China

${ }^{8}$ Department of Nephrology, Beijing Friendship Hospital Attached Capital Medical University, Beijing, China

${ }^{9}$ Department of Nephrology, West China Hospital of Sichuan University, Chengdu, China

${ }^{10}$ Department of Nephrology, Zhongshan Hospital, Fudan University, Shanghai, China

${ }^{11}$ Department of Nephrology, Dalian Municipal Central Hospital, Dalian, China

${ }^{12}$ Department of Nephrology, The First Affiliated Hospital, Sun Yat-sen University,

Guangzhou, China

${ }^{13}$ Department of Nephrology, General Hospital of Shenyang Military Region,

Shenyang, China

${ }^{14}$ Department of Nephrology, General Hospital of Ningxia Medical University,

Yinchuan, China

${ }^{15}$ Department of Nephrology, Renji Hospital Affiliated to Shanghai Jiao Tong University School of Medicine, Shanghai, China

${ }^{16}$ Department of Nephrology, Huashan Hospital, Fudan University, Shanghai, China

${ }^{17}$ Department of Nephrology, Xijing hospital of The Fourth Military Medical

University, Xi'an, China

${ }^{18}$ Department of Nephrology, Guangdong General Hospital, Guangzhou, , China

${ }^{19}$ Department of Nephrology, General Hospital of Benxi Iron and Steel Co., Ltd,

Benxi, China

${ }^{20}$ Department of Nephrology, Daping Hospital Affiliated to Army Military Medical University, Chongqing, China

${ }^{21}$ Department of Nephrology, Affiliated Zhong Shan Hospital of Dalian University, Dalian, China

${ }^{22}$ Department of Nephrology, Lanzhou University Second Hospital, Lanzhou, China

${ }^{23}$ Department of Nephrology, The First Affiliated Hospital of Harbin Medical University, Harbin, China

${ }^{24}$ Department of Nephrology, The First Affiliated Hospital of Xinjiang Medical University, Urumchi, China

${ }^{25}$ Department of Nephrology, Zhejiang Provincial People's Hospital, Hangzhou, , China

${ }^{26}$ Department of Nephrology, An Steel Group Hospital, Anshan, China

${ }^{27}$ Department of Nephrology, The People's Hospital of Zhengzhou University and Henan Provincial People's Hospital, Zhengzhou, China

${ }^{28}$ Department of Nephrology, The Second Affiliated Hospital of Harbin Medical University, Harbin, China

${ }^{29}$ Department of Nephrology, General Hospital of Mining Industry Group FuXin,

FuXin, China

${ }^{30}$ Department of Nephrology, The First Hospital of China Medical University, Shenyang, China

${ }^{31}$ Department of Nephrology, General Hospital Affiliated To Shenyang Medical College, Shenyang, China

${ }^{32}$ Department of Nephrology, Shengjing Hospital of China Medical University, Shenyang, China

${ }^{33}$ FibroGen, Inc, San Francisco, California, USA

Acknowledgements The authors thank all staff for their support with this study and the members of the patient and public involvement group for their invaluable advice. We acknowledge Dr. Mark Roger Marshall (Associate Professor of University of Auckland, New Zealand) for his expert guidance and helpful comments on the study design.

Funding The ADIFE study is an investigator-initiated and conducted study. This work is supported by the special fund for National Health and Family Planning Commission (NHFPC) Scientific Research in the Public Welfare (grant number 201502023). The NHFPC will have no role in the design, conduct, management, analysis, or interpretation of the study.

Competing interests None declared.

Patient consent for publication Parental/guardian consent obtained.

Provenance and peer review Not commissioned; externally peer reviewed.

Open access This is an open access article distributed in accordance with the Creative Commons Attribution Non Commercial (CC BY-NC 4.0) license, which permits others to distribute, remix, adapt, build upon this work non-commercially, and license their derivative works on different terms, provided the original work is properly cited, appropriate credit is given, any changes made indicated, and the use is non-commercial. See: http:// creativecommons.org/licenses/by-nc/4.0/.

\section{REFERENCES}

1. Woo K-T, Choong HL, Wong K-S, et al. The contribution of chronic kidney disease to the global burden of major noncommunicable diseases. Kidney Int 2012;81:1044-5.

2. Feng $L$, Jin AZ, Allen JC, et al. Timing of commencement of maintenance dialysis and mortality in young and older adults in Singapore. BMC Nephrol 2017;18:176.

3. Park JY, Yoo KD, Kim YC, et al. Early dialysis initiation does not improve clinical outcomes in elderly end-stage renal disease patients: a multicenter prospective cohort study. PLoS One 2017;12:e0175830.

4. Mehrotra R, Rivara M, Himmelfarb J. Initiation of dialysis should be timely: neither early nor late. Semin Dial 2013;26:644-9.

5. Churchill DN. An evidence-based approach to earlier initiation of dialysis. Am J Kidney Dis 1997;30:899-906.

6. Traynor JP, Simpson K, Geddes CC, et al. Early initiation of dialysis fails to prolong survival in patients with end-stage renal failure. $J \mathrm{Am}$ Soc Nephrol 2002;13:2125-32.

7. Faust $J$, Schreiner $O$. Early versus late initiation of dialysis. $N$ Engl $J$ Med 2010;363:2368-9.

8. Wingard RL, Pupim LB, Krishnan M, et al. Early intervention improves mortality and hospitalization rates in incident hemodialysis patients: RightStart program. Clin J Am Soc Nephrol 2007;2:1170-5.

9. Korevaar JC, Jansen MAM, Dekker FW, et al. When to initiate dialysis: effect of proposed us guidelines on survival. The Lancet 2001;358:1046-50.

10. Cooper BA, Branley P, Bulfone L, et al. A randomized, controlled trial of early versus late initiation of dialysis. $N$ Engl $J$ Med 2010;363:609-19.

11. Wright $\mathrm{S}$, Klausner D, Baird B, et al. Timing of dialysis initiation and survival in ESRD. Clin J Am Soc Nephrol 2010;5:1828-35.

12. Hwang S-J, Yang W-C, Lin M-Y, et al. Impact of the clinical conditions at dialysis initiation on mortality in incident haemodialysis patients: a national cohort study in Taiwan. Nephrology Dialysis Transplantation 2010;25:2616-24.

13. Susantitaphong P, Altamimi S, Ashkar M, et al. Gfr at initiation of dialysis and mortality in CKD: a meta-analysis. Am J Kidney Dis 2012;59:829-40.

14. Zaman T, Filipowicz R, Beddhu S. Implications and importance of skeletal muscle mass in estimating glomerular filtration rate at dialysis initiation. J Ren Nutr 2013;23:233-6.

15. Escoli R, Luz I, Santos P, et al. Glomerular filtration rate and initiation of dialysis. Ther Apher Dial 2017;21:606-10.

16. Lin X, Zeng X-Z, Ai J. The glomerular filtration rate (GFR) at dialysis initiation and mortality in chronic kidney disease (CKD) in East Asian populations: a meta-analysis. Intern Med 2016;55:3097-104.

17. Lassalle M, Labeeuw M, Frimat L, et al. Age and comorbidity may explain the paradoxical association of an early dialysis start with poor survival. Kidney Int 2010;77:700-7.

18. Brown MA, Collett GK, Josland EA, et al. Ckd in elderly patients managed without dialysis: survival, symptoms, and quality of life. Clin J Am Soc Nephrol 2015;10:260-8.

19. National Kidney F. KDOQI clinical practice guideline for hemodialysis adequacy: 2015 update. Am J Kidney Dis 2015;66:884-930.

20. Grootendorst DC, Michels WM, Richardson JD, et al. The MDRD formula does not reflect GFR in ESRD patients. Nephrology Dialysis Transplantation 2011;26:1932-7.

21. Liu $Y$, Wang $D$, Chen $X$, et al. An equation based on fuzzy mathematics to assess the timing of haemodialysis initiation. Sci Rep 2019;9:5871.

22. Chan A-W, Tetzlaff JM, Altman DG, et al. Spirit 2013 statement: defining standard protocol items for clinical trials. Rev Panam Salud Publica 2015;38:506-14

23. Levey AS, Stevens LA, Schmid $\mathrm{CH}$, et al. A new equation to estimate glomerular filtration rate. Ann Intern Med 2009;150:604-12.

24. Taler SJ, Agarwal R, Bakris GL, et al. KDOQI us commentary on the 2012 KDIGO clinical practice guideline for management of blood pressure in CKD. Am J Kidney Dis 2013;62:201-13.

25. Kliger AS, Foley RN, Goldfarb DS, et al. KDOQI us commentary on the $2012 \mathrm{KDIGO}$ clinical practice guideline for anemia in CKD. Am J Kidney Dis 2013;62:849-59.

26. Isakova T, Nickolas TL, Denburg M, et al. KDOQI US Commentary on the 2017 KDIGO Clinical Practice Guideline Update for the Diagnosis, Evaluation, Prevention, and Treatment of Chronic Kidney Disease-Mineral and Bone Disorder (CKD-MBD). Am J Kidney Dis 2017;70:737-51.

27. Group CNAHAC. Clinical practice guide for Chinese hemodialysis adequacy. Natl Med J China 2015;95:2748-53.

28. Chen XM, Ding XQ, Liu FY, et al. Blood Purification Standard Operating Procedure(SOP):2010 version, 2010.

29. Raguso CA, Maisonneuve N, Pichard C. [Subjective Global Assessment (SGA): evaluation and followup of nutritional state]. Rev Med Suisse Romande 2004;124:607-10. 
30. Hays RD, Kallich JD, Mapes DL, et al. Development of the kidney disease quality of life (KDQOL) instrument. Qual Life Res 1994;3:329-38.

31. Hays RD KJ, Mapes D, Coons S, et al. Kidney disease quality of life short form (KDQOL-SFTM), version 1.3: a manual for use and scoring.

32. Nasreddine ZS, Phillips NA, Bédirian V, et al. The Montreal cognitive assessment, MoCA: a brief screening tool for mild cognitive impairment. J Am Geriatr Soc 2005;53:695-9.

33. Mansournia MA, Higgins JP, Sterne JA, et al. Biases in randomized trials: a conversation between Trialists and epidemiologists. Epidemiology 2017;28:54-9.
34. Mansournia MA, Altman DG. Inverse probability weighting. BMJ 2016;352.

35. Pan Y, Xu XD, Guo LL, et al. Association of early versus late initiation of dialysis with mortality: systematic review and meta-analysis. Nephron Clin Pract 2012;120:c121-31.

36. Yamagata K, Nakai S, Iseki K, et al. Late dialysis start did not affect long-term outcome in Japanese dialysis patients: long-term prognosis from Japanese Society for [corrected] Dialysis Therapy Registry. Ther Apher Dial 2012;16:111-20.

37. Scialla JJ, Liu J, Crews DC, et al. An instrumental variable approach finds no associated harm or benefit with early dialysis initiation in the United States. Kidney Int 2014;86:798-809. 